The response to $\mathrm{K}^{+}$replacement was dramatic and the patient was able to walk the following day. He was sent home on oral thyroxin $100 \mu$ g daily. $\mathrm{K}^{+}$supplements were omitted subsequently as the serum $\mathrm{K}^{+}$was normal. Thyroid functions improved and he did not develop any further paralytic attacks.

\section{Discussion}

This patient had features suggestive of HPP without evidence of $\mathrm{K}^{+}$loss through the kidneys or the gut. The recurrent episodes of hypokalemia could most likely be due to intracellular shift of $\mathrm{K}^{+}$. Most forms of HPP are genetic, with autosomal dominant inheritance. These diseases are channelopathies, (caused by mutations in ion channel genes). The culprit genes encode muscle sodium (SCN4A), calcium (CACNA1S) and potassium (KCNE3) channels [2, 3]. Thyrotoxic hypokalemic periodic paralysis is a well-established phenomenon associated with HPP but our patient had subclinical hypothyroidism with autoimmune thyroiditis. A similar case was reported in Japan 1990 of recurrent hypokalemic paralytic attacks associated with hypothyroidism due to autoimmune thyroiditis. The patient had no further attacks following treatment with thyroxine [4].

Our patient had EMG evidence of neuromyotonia, which is a condition characterised by hyperexcitability of peripheral nerves manifesting as continuous muscle fibre activity. Neuromyotonia is well known to be associated with other auto immune diseases. Patients harbour antibodies directed against voltage-gated $\mathrm{K}^{+}$channels. These antibodies reduce the $\mathrm{K}^{+}$current conducted by these channels and thus lead to a prolongation of the nerve action potential resulting in increased neurotransmitter release, manifesting clinically as muscle hyperexcitability [5].

This patient with subclinical hypothyroidism and autoimmune thyroiditis had HPP with EMG evidence of neuromyotonia which are diseases both related to $\mathrm{K}^{+}$ channels. This raises the possibility of a transient autoimmune process causing antibodies against $\mathrm{K}^{+}$channels causing this patients disease manifestations. Detailed genetic and antibody studies for confirmation of channelopathy and neuromyotonia respectively were not performed due to non availability of these investigations in the country.

\section{References}

1. Ahlawat SK, Sachdev A. Hypokalaemic paralysis. Postgraduate Medical Journal 1999; 75: 193-7.

2. Fontaine B. Periodic paralysis: new pathophysiological aspects. Bulletin del' Academie Nationale de Med 2008; 192: $1543-8$.

3. http://neuromuscular.wustl.edu/mtime/mepisodic.html

4. Kinoshita I, Usa T, Satoh A, Tsujihata M. A case of hypothyroidism associated with hypokalaemic periodic paralysis. Rinsho Shinkeigaku 1990; 30: 100-2 (Japanese).

5. M. Benatar. Neurological potassium channelopathies. Quarterly Journal of Medicine 2000; 93: 787-97.

\title{
A case of warm autoimmune haemolytic anaemia with intravascular haemolysis: a rare presentation
}

\section{Gunawardena ${ }^{1}$, G A C Gamakaranage ${ }^{2}$}

Ceylon Medical Journal 2013; 58: 176-7

\section{Introduction}

Autoimmune haemolytic anaemia (AIHA) is a rare (prevalence $0.2-1.0$ per 100,000 population) but a potentially fatal condition [1]. Diagnosis of AIHA is based mainly on Direct Agglutination Test (DAT/Coomb’s test) [2]. AIHA is classified into warm (optimal autoantibody reactivity at $37^{\circ} \mathrm{C}$ ) and cold (optimal autoantibody reactivity at $4^{\circ} \mathrm{C}$ ) types. Based on the presence or absence of an underlying cause, it is divided into primary (idiopathic) and secondary (secondary to lympho proliferative disorders, autoimmune disease, drugs and non-haematological malignancies).

Departments of ${ }^{1}$ Pathology and ${ }^{2}$ Clinical Haematology, Faculty of Medical Sciences, University of Sri Jayewardenepura, Sri Lanka.

Correspondence: DG, e-mail: <dammika.g@hotmail.com>. Received 7 September 2013 and revised version accepted 23 February 2013. Competing interests: none declared. 
Warm type accounts for about $70 \%$ of cases with AIHA [3]. It can occur at any age, but mostly after 40 years [4].

Haemolysis in warm AIHA is mediated by IgG alone or IgG with complement. Once immunoglobulins are bound, the red cells are taken up by the macrophages of the reticulo-endothelial system which has receptors for the Fc fragments of the immunoglobulins. As the red cells are haemolysed extravascularly this occurs mainly in the spleen. Intravascular haemolysis is unusual in warm AIHA, although it can occur rarely, as warm autoantibodies can fix complements [3,4].

We report severe warm AIHA in an adult with evidence of intravascular haemolysis, which is an unusual presentation.

\section{Case report}

A 37-years old mother of two children was seen in the haematology clinic, for investigation of anaemia, three months after her second delivery. She complained of fatigue and headache. She was otherwise asymptomatic. The anaemia was detected during the antenatal period of her second pregnancy at a routine checkup. She had diabetes mellitus and hypothyroidism of 10 years duration. She had undergone thyroidectomy for Hashimoto's thyroiditis and was treated with thyroxin and metformin. She did not have a significant family history of illness or recurrent blood transfusions. She was pale, icteric and afebrile. There was a tender hepatomegaly $(4 \mathrm{~cm})$ but no splenomegaly or lymphadenopathy.

When she was seen in the ward, the haemoglobin was $6.2 \mathrm{~g} / \mathrm{dl}$. She had a normal platelet count of $280,000 \times 10^{9} / 1$ with a neutrophil leucocytosis. Reticulocyte count was $17.6 \%$ indicating haemolysis. The blood picture showed spherocytes, normochromic normocytic and some hypochromic microcytic red cells, occasional red cell aggregates, nucleated red cells and polychromatic cells (Figure 1). She had an indirect hyperbilirubinaemia of $38.6 \mu \mathrm{mol} / 1$, with slightly elevated liver transaminases. The direct antiglobulin test was strongly positive for $\mathrm{C} 3 \mathrm{~d}$ and IgG both, but negative for IgM. The test for haemosiderinuria was strongly positive indicating a chronic intravascular haemolysis (Figure 2). The saline agglutination test showed a few red cell clumps at $4{ }^{\circ} \mathrm{C}$ and occasional clumps at room temperature. The cold antibody titre was insignificant at temperatures $4^{\circ} \mathrm{C}$ to $30^{\circ} \mathrm{C}$ favouring a warm antibody. Mycoplasma and Donath Landsteiner antibodies were negative. Ultrasonically, there was mild hepatomegally and splenomegally $(14 \mathrm{~cm})$ with no paraaortic lymphadenopathy. The antinuclear antibody was negative. TSH was slightly low. Serum iron studies were normal. Subsequently a bone marrow examination was done and it showed only an erythroid hyperplasia with no abnormal cell infiltrates.

As she was symptomatic with a haemoglobin of $6.2 \mathrm{~g} /$ $\mathrm{dl}$ and three units of packed red cells were given. It was difficult to achieve a remission (normal haemoglobin with no haemolysis), even following several blood transfusions and steroid therapy. However, after about six months of steroid therapy, the haemoglobin became normal. Currently she is in remission with no relapses. However she needs an intermittent, small dose of steroids to maintain a sustained remission over the years of follow up.

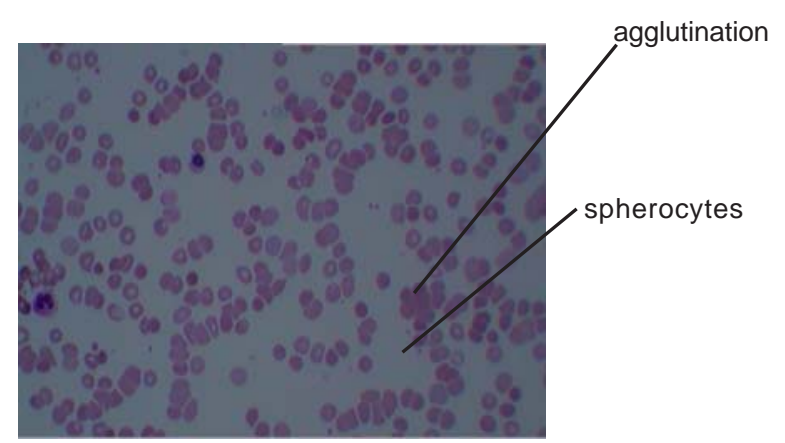

Figure 1. Blood picture showing spherocytes with agglutination.

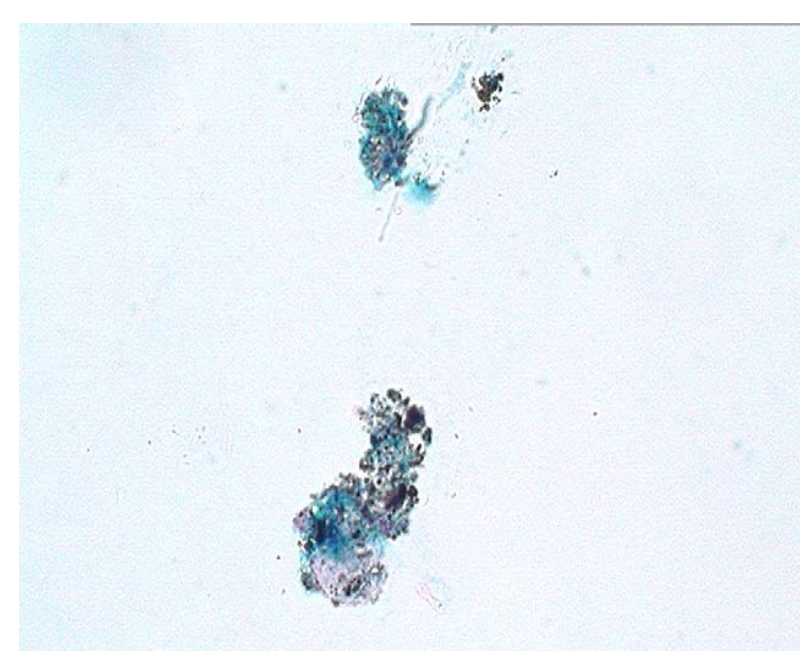

Figure 2. Urine for haemosiderin - strongly positive.

\section{Discussion}

Warm autoimmune haemolytic anaemia which is the commoner type of AIHA, can occur at all ages but mostly above 40 years [4]. The clinical presentation may range from insidious onset to acute or life threatening fulminating haemolysis [3]. The haemolysis is mediated by an $\mathrm{IgG}$ antibody which reacts optimally at $37^{\circ} \mathrm{C}$. The haemolysis is mainly extravascular, primarily in the spleen and to some extent in the liver. Although many warm antibodies fix complement, intravascular complement mediated haemolysis and haemoglobinuria are unusual in warm AIHA [4]. Complement mediated intravascular haemolysis can rarely occur in warm AIHA, resulting in hamoglobinuria and haemosiderinuria [3]. Autoagglutination of red cells 
can occur in some patients with warm AIHA, which can be differentiated from cold autoagglutination that occurs in cold AIHA by the reversal of agglutination by warming to $37^{\circ} \mathrm{C}$ in the latter, but not in the warm type [5].

Investigations showed that the patient had severe haemolytic anaemia on presentation, the haemoglobin was $6.2 \mathrm{~g} / \mathrm{dl}$, with a positive haemolytic screen of reticulocytosis, indirect hyperbilirubinaemia and erythroid hyperplasia in the bone marrow. According to the blood picture it is a spherocytic anaemia and the positive DAT indicates that it is immune mediated. Since it is positive for both C3d and IgG, it is considered as warm autoimmune hemolytic anaemia. However, subsequently her urine was strongly positive for haemosiderin which showed evidence of intravascular haemolysis.

\section{References}

1. Crowther M, Chan YL, Garbett IK, et al. Evidence based focused review of the treatment of Idiopathic Warm haemolytic anaemia in adults. Blood 2011; 118: 4036-40.

2. Michel M, Classification and therapeutic approaches in autoimmune haemolytic anaemia: an update. Expert Review of Hematology 2011; 4: 607-18.

3. Hoffbrand AV, Catovsky D, Tudenham EGD. Postgratuate Haematology; 6th Edition Wiley- Blackwell Ltd.

4. Packman $\mathrm{CH}$, Haemolytic anaemia due to warm autoantibodies. Blood Reviews 2008; 22: 17-31.

5. Petz LD. Cold antibody autoimmune haemolytic anaemias. Blood Reviews 2008; 22: 1-15.

\title{
A patient with severe body aches without fever progressing to dengue shock syndrome
}

\author{
R Premaratna $^{1}$, J K N D Miththinda ${ }^{2}$, C Abeyrathna ${ }^{2}$, H J de Silva ${ }^{1}$
}

Ceylon Medical Journal 2013; 58: 178-9

\section{Introduction}

More than $80 \%$ of dengue infections are asymptomatic, only 5\% develop more severe illness and an even smaller proportion develops life-threatening complications $[1,2]$. Characteristically dengue presents as an acute febrile illness associated with headache, myalgia, arthralgia and a rash, and typically goes through three phases; febrile, critical and recovery [2]. Several atypical manifestations of dengue such as neurological, cardiac, gastrointestinal, respiratory and renal have been reported [3].

We report a case of dengue shock syndrome (DSS), presenting with myalgia and arthralgia, but no history of fever.

\section{Case report}

A 23-year old previously healthy male with three days of body aches, headache, lethargy and nausea, but no documented fever was admitted to hospital due to insistence of the patient and relatives. There was no abdominal pain, vomiting or bleeding manifestations. He had self-medicated with paracetamol $1 \mathrm{~g}$ on an irregular basis for body aches. He had not consumed NSAIDs or steroids.

He was dark in complexion. He was afebrile, pulse rate was 98 beats /minute regular and the blood pressure (BP) was 110/70 mmHg in the sitting position. There was 4 $\mathrm{cm}$ tender hepatomegaly but no splenomegaly or free fluid in the abdomen. The chest and the central nervous system examination were normal.

The following day (day five of the illness) he developed postural dizziness and became drowsy, the BP was 90/60 $\mathrm{mmHg}$ and pulse rate was100/minute. Resuscitation was carried out with IV crystalloid boluses. He remained afebrile but the body aches persisted. On day six of the illness he developed sub conjunctival haemorrhages, gum bleeding and a right sided pleural effusion. The platelet count dropped to $3 \times 10^{9} / \mathrm{l}$, $\mathrm{Hb}$ rose $16 \%$ and PCV $13 \%$ and the AST rose to $251 \mathrm{iu} / \mathrm{l}$. At this point he developed the only documented fever spike of $101^{\circ} \mathrm{F}$. Dengue Ig M antibodies were positive.

Departments of ${ }^{1}$ Medicine and ${ }^{2}$ Public Health, Faculty of Medicine, University of Kelaniya, Sri Lanka.

Correspondence: RP, e-mail: <ranjanp64@gmail.com>. Received 8 January 2013 and revised version accepted 16 June 2013. Competing interests: none declared. 